\title{
Evanescent luminescence and nanometer-size light source
}

\author{
Raoul Kopelman ${ }^{\mathrm{a}}$, Klonimus Lieberman ${ }^{\mathrm{b}}$, Aaron Lewis ${ }^{\mathrm{b}}$ and Weihong Tan ${ }^{\mathrm{a}}$ \\ a Department of Chemistry, University of Michigan, Ann Arbor, MI 48109, USA \\ b Department of Applied Physics, Hebrew University, Jerusalem 91904, Israel
}

\begin{abstract}
Subwavelength light sources have been constructed with the aid of luminescent materials. These EXCITOR (exciton transmitted optical radiation) sources are of both theoretical and practical interest. The production of evanescent luminescence may test some of the predictions of quantum electrodynamics concerning the interactions of matter and radiation (e.g., when the emission process and the "subsequent" absorption process are no longer independent). Furthermore, the scannable luminescent tip can be applied to near-field optical microscopy, to scanning exciton microscopy, and to sub-microspectroscopy. An example of an EXCITOR consists of a gold-plated glass micropipette with an inner diameter tapering down to 50 nanometer, "plugged" with an anthracene microcrystal or a polymeric matrix doped with a laser dye. Design considerations involve optical, excitonic, photochemical, and mechanical properties of the luminescent point source. In the ideal limit, the luminescent source consists of a single active chromophore (analogous to the photosynthetic reaction center). As it is scanned over a sample, it senses a variety of perturbations on the atomic or molecular scale such as quenching or external heavy atom effect.
\end{abstract}

\section{Introduction}

In conventional "far-field" light imaging, the image cannot be smaller than the diffraction limit, roughly $\lambda / 2$, where $\lambda$ is the wavelength [1]. In this case it is irrelevant whether the source is a laser beam, an ultrasmall lamp $(\leqslant \lambda / 2)$ or a simple luminescent center of atomic size. However, in "near-field" applications, the size of the image is only limited by the size of the source $(a)$ and its distance $(d)$ [2,3]. If $d<a$, the image size is roughly equal to the source size $(a)$. At this point the following questions become relevant: (1) What is the lowest limit for the source size $a$ ? (2) What is the brightness of such a source? (3) How stable is its position? (4) Can it be scanned? (5) How stable is the scanning? (6) How stable is the light intensity? (7) What are its spectral characteristics? (8) How robust is it mechanically and chemically?

There is another set of questions: What is the nature of the "image"? Does it involve photons, evanescent photons, or energy $(h \nu)$ packages? Can we at $a=d<\lambda$ distinguish an emission plus absorption event from an energy transfer event? Does it matter in practice? What is the detection sensitivity? What is the spectral resolution? Can one fully separate the nature of the target from the nature of the source or do they form a coupled system?

\section{Theoretical considerations}

The simplest nanometer light source is a nanometer sized hole, stopping down light from a larger light source, e.g., a laser. For the sake of contrast, the hole's surrounding must be totally opaque. The thinnest matrix for such a hole is typically aluminum, the metal with the shortest extinction length $(6 \mathrm{~nm})$. It is opaque enough for good contrast at a thickness of $50 \mathrm{~nm}$ [3].

The thickness $(b)$ of the plate is effectively the length $(l)$ of the tunnel defined by the hole (aperture $a$ ). For $a<\lambda$, the probability $P$ of a photon to pass through the tunnel falls off as

$P \sim a^{6} \exp (-3.6 l / a)$.

Obviously, when $a=l / 10$ instead of $a=l$, this reduces the throughput by about $10^{22}$. However, for a given size $a \approx \lambda$, there will be significantly 
more throughput if the effective $\lambda$ shrinks by a factor of 2 or more (16 times or more). This can be achieved, in principle, by filling the tunnel with a transparent material of high refractive index $n$ (since $\lambda=\lambda_{0} / n$, where $\lambda_{0}$ is the wavelength in vacuum), or a high effective $n$ (e.g. near a strong absorption edge).

To overcome the exponential decrease of light intensity with $a$, we transform the point source from a passive aperture to an active emitter, e.g. anthracene crystal fluorescence [4]. The submicron tip of a metal-coated glass micropipette is filled with the molecular crystal (fig. 1). The incoming photons propagate through the super-micron portion of the pipette and get absorbed by the crystal (the back-mirrored glass walls act as a light pipe). The absorbed photons generate Frenkel excitons that propagate (diffuse) towards the submicron tip (the glass walls may also serve as an effective light pipe). We note here that the diffusion length of anthracene is somewhat controversial. While the old data $[5,6]$ give about $500 \AA(0.05 \mu \mathrm{m}$, perpendicular to the $a b$ plane) the more recent, roomtemperature data [7] give about $10 \mu \mathrm{m}$, e.g., 200 times longer. Even if the latter data are contami-

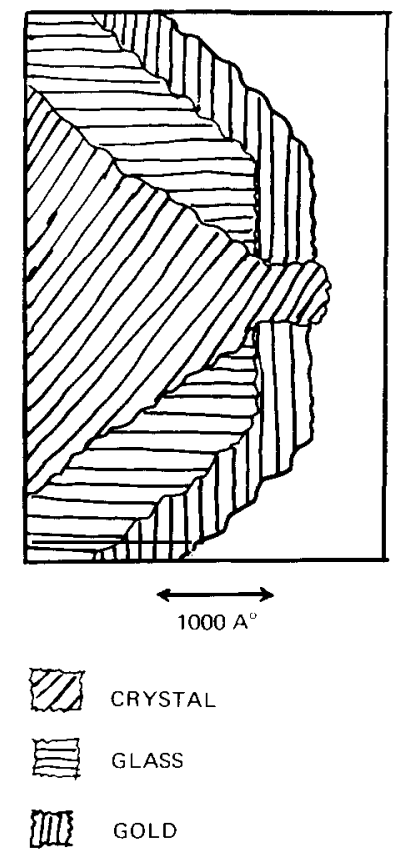

Fig. 1. Schematic drawing of EXCITOR tip. nated by some emission-reabsorption events (radiative transport), this does not matter much from our point of view. Due to the overlap of the absorption and the emission spectra at room temperature (hot bands), the reabsorption process is fairly efficient (and includes some emitted light returning from the glass walls). The main point is that the exciton transport is largely controlled by the geometry of the crystal, i.e. the geometry of the tip's cavity. Thus, the efficiency decreases geometrically, rather than exponentially, with smaller aperture size. With a quantum efficiency of nearly unity, the major loss of photons is by fluorescence in the back-direction rather than forward direction. The forward/backward ratio of these two fluxes should be proportional to the ratio of cross-sections. If the diameter of the crystal at the back is 0.5 and in front 0.05 microns then this forward/backward efficiency should be 1/100. Another loss channel is the exciton-plasmon transfer to the metal (gold) ring at the tip. This reduces the effective tip radius by about the critical distance (defined below), which is about $0.005 \mu \mathrm{m}$.

Of particular interest is the very near-field interaction between the exciton at the tip and the sample. A metallic sample will quench the exciton with a typical critical distance $d_{\mathrm{s}}$, defined as the distance at which this quenching probability is 0.5 (reducing the lifetime by a factor of two). Similarly, such a Förster-Kuhn-Drexhage distance [8,9] also describes the quenching by a dielectric film of quenchers. However, in the latter case, the quenching acceptors can also be efficient fluorophores.

How critical is exciton-exciton annihilation? The annihilation rate $R$ is related to the density $\rho$ quadratically:

$R=k \rho^{2}, \quad k \sim D$,

where $D$ is the diffusion constant. However, for dimensions smaller than the diffusion length (and with reflective boundary conditions) this changes. For a one-dimensional wire (with diameter less than the diffusion length) one has [12]:

$R^{\prime}=k^{\prime} \rho^{3}, \quad k^{\prime} \sim D$,

giving

$R^{\prime} / R \approx \rho$. 
Thus, to maintain a steady state and keep up with annihilation, a much lower photon flux is required for a one-dimensional crystal, compared to a threedimensional one. A typical exciton number density (per site) may be $10^{-4}$, giving a significant reduction factor of $R^{\prime} / R \approx 10^{-4}$. We note that for a thicker wire, or one with a conical shape, with a diameter close to the diffusion length, an approximate relation may be $R^{\prime} \sim \rho^{2.5}$. This would give $R^{\prime} \sim \rho^{0.5}$ or $R^{\prime} / R \sim 10^{-2}$ for $\rho=10^{-4}$. On the other hand, the trapping rate $R_{\mathrm{T}}$ will be linear in $\rho$ for all geometries (but not linear in the trap concentration). Thus an arrangement utilizing trapping or quenching at the narrow tip will be more competitive with annihilation for smaller crystalline domains. Obviously, the fluorescence rate $R_{\mathrm{F}}$ is also linear with $\rho$. Whether one wants to optimize fluorescence or trapping, increasing the laser photon flux may quickly reach the limit of diminishing returns.

For biological and chemical analysis, the sensitivity to a single chromophore or fluorophore molecule in the sample is of particular interest. If the tip diameter is of the order of the Förster radius or the Förster-Kuhn-Drexhage critical distance, and the sample distance (including the fluorophore) is the same, the probability of detecting this molecule is extremely high (requiring just a few excitons at the tip). We note that for a single highly efficient fluorophore (very high absorption coefficient and quantum efficiency near unity) about $10^{9}$ photons are required $[4,10,11]$ to pass through such a sample (with a $\lambda / 2$ distance from the molecule) to generate one fluorescence quantum (photon). Will it also take $10^{9}$ photons to create the required few excitons? Theoretically it will take only $10^{4}$ to $10^{5}$ photons with a single crystal. Furthermore, if the crystal surface is covered with appropriate "exciton traps" that act as donors for the fluorophore, the host crystal will serve as an antenna. This effective antenna could easily consist of $10^{6}$ or $10^{7}$ host molecules. Thus only about $10^{3}$ or less photons will be required in this case. Such a reduced photon flux will significantly reduce linear or non-linear effects and thus may reduce local heating, chemical decomposition, or Raman emission. Furthermore, not only will the single fluorophore be detected non- destructively, but its location will also be established to within the Förster radius (e.g., 25 or $50 \AA$ ).

The photodestruction (degradation) of the fluorescent species (dye) is an important aspect $[11,12]$. The rate $\left(\mathrm{s}^{-1}\right)$ of photodestruction is given by

$k_{\mathrm{d}}=\sigma_{\mathrm{a}} I \phi$,

where $\sigma_{\mathrm{a}}$ is the absorption cross section $\left(\mathrm{cm}^{2} /\right.$ molecule $), I$ the incident beam intensity (photons $/ \mathrm{cm}^{2} \mathrm{~s}$ ) and $\phi$ the quantum yield of photodestruction [11]. For dyes in solution $\phi=$ $2.7 \times 10^{-5}$ for fluorescein and $1.1 \times 10^{-5}$ for Bphycoerythrin [11]. However, in solid matrices this can be reduced to about $10^{-10}$ [13]. This involves optimization and purification of dye, matrix and solvent, as well as the addition of triplet quenchers and the use of barrier films to reduce the gaseous exchange of oxygen and water [13]. We also note that multiphoton or multiquanta destruction processes of the emitting "supertrap" can be largely circumvented by back-transfer into the host antenna. Thus the reduction of the photodestruction efficiency is as much a molecular engineering job as is the enhancement of the EXCITORs quantum efficiency and the scaling down of its effective size.

\section{Experimental}

The hole sizes at the tip of gold and aluminum coated pipettes were determined by SEM (figs. $2,3)$ and had diameters down to $50 \mathrm{~nm}$. For some pipettes, anthracene crystals were grown first and then gold-coated. The crystals were seen to protrude slightly, having a disk shape (thus proving their extension throughout the tip). Crystals of anthracene, DCM, l-amino-anthracene, perylene, pyrene, diphenyl-anthracene and tetracene have been grown. Some details have been given earlier [4]. The excitation was with a $20 \mathrm{~W}$ argon ion laser (cw), using mostly the $360 \mathrm{~nm}$ lines, but occasionally the blue line (for tetracene).

Preliminary amplification factors for anthracene tips have been given earlier $[4,10]$. The order of magnitude amplification factors have also been found for 50 and $100 \mathrm{~nm}$ tips of various crystals, e.g.DCM[4-(dicyano-methylene ) - 2-methyl-6-( $\rho$ - 


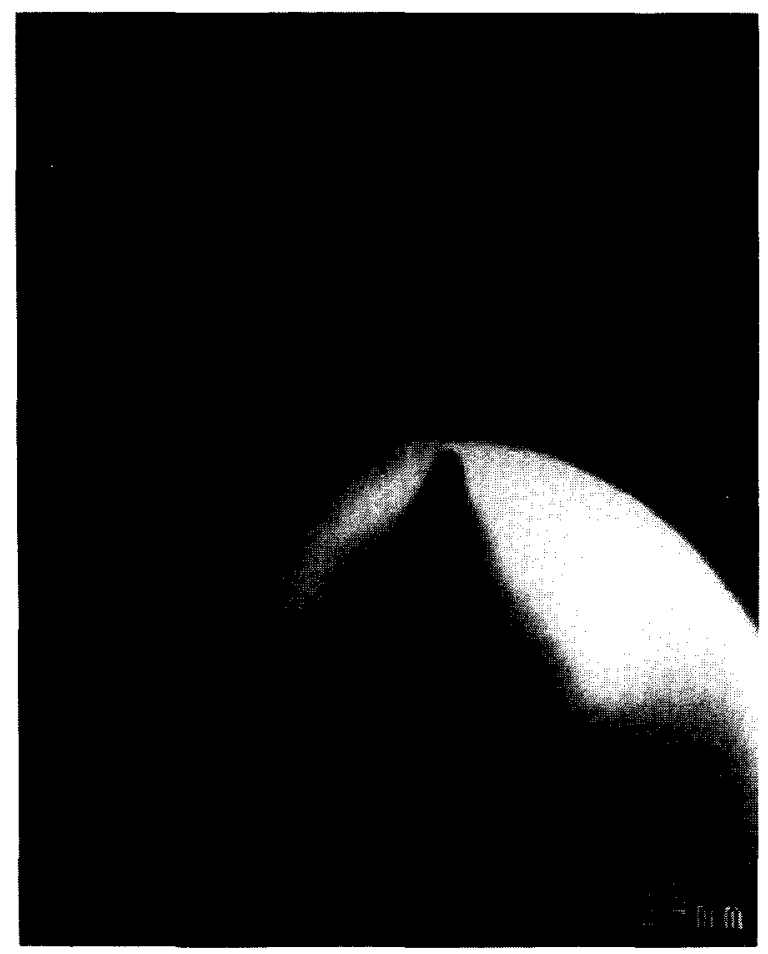

Fig. 2. Low resolution SEM scan of gold-plated micropipette tip.

dimethylamino-styryl)-4H-pyran], using monochromatized Xe lamp excitation as well as the argon ion laser green line. We mention particularly the red-emitting crystals, where amplification was measured relative to the UV exciting photons. The red light's wavelength inside the crystal is still longer than the UV exciting light's wavelength in air, showing that the amplification goes well beyond the index of refraction effect $(n \approx 1.5)$.

The extension of the anthracene crystal throughout the tip (see above) has also been shown by exciton quenching experiments. A $20 \mathrm{~nm}$ gold film (transparent to blue light) on glass is $Z$ scanned by the anthracene tip. Only when the distance is below $10 \mathrm{~nm}$ is there significant reduction in the light intensity transmitted by this gold "mirror".

\section{RAMETIIE}

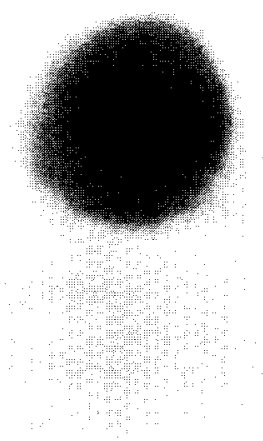

Fig. 3. High resolution SEM scan of gold-plated micropipette tip (ID about $120 \mathrm{~nm}, \mathrm{OD}$ about $550 \mathrm{~nm}$ ).

\section{Acknowledgement}

Supported by NSF grant DMR-8801120 and DOE grant DE-FG02-90ER60984 (R.K. and W.T.) and by the Innovative Science and Technology Office of the Strategic-Defense Initiative administered by the Office of Naval Research and the Israeli National Council for Research and Development (A.L. and K.L.).

\section{References}

[1] E. Abbe, Archiv. Mikroskop. Anat. 9 (1873) 413.

[2] J.A. O'Keefe, J. Opt. Soc. Am. 46 (1956) 359.

[3] E. Betzig, A. Harootunian, A. Lewis and M. Issacson, Appl. Opt. 25 (1986) 1890. 
[4] S. Lieberman, S. Harush, A. Lewis and R. Kopelman, Science 247 (1990) 59.

[5] V.M. Agranovich and M.D. Galanin, Electronic Excitation Energy Transfer in Condensed Matter (NorthHolland, Amsterdam, 1982).

[6] D. Donati and J.O. Williams, Mol. Cryst. Liq. Cryst. 44 (1978) 23.

[7] H. Nishimura, T. Yamaoka, K. Hattori, A. Matsui and K. Mizuno, J. Phys. Soc. Jpn. 54 (1985) 4370.

[8] K.H. Drexhage, J. Lumin. 1 \& 2 (1970) 693.

[9] K.H. Drexhage, in: Progress in Optics, ed. E. Wolf, (North-Holland, Amsterdam, 1974) p. 163.
[10] R. Kopelman, A. Lewis and K. Lieberman, J. Lumin. 45 (1990) 298.

[11] R.A. Mathies and L. Stryer, in: Applications of Fluorescence in the Biomedical Sciences, eds., D.L. Taylor, A.S. Waggoner, R.F. Murphy, F. Lanny and R.R. Birge (Liss, New York, 1986) p. 129.

[12] R. Kopelman, Science 241 (1988) 1620.

[13] P.S. Friedman and C.R. Parent, Solar Energy Research Inst. Report, STR-211-3149 (DE87001172) (Golden, CO, 1987). 